\title{
COMPARISON OF ZEB1 AND LEICA C10 INDOOR LASER SCANNING POINT CLOUDS
}

\author{
Beril Sirmacek $^{a *}$, Yueqian Shen $^{a}$, Roderik Lindenbergh $^{a}$, Sisi Zlatanova $^{b}$, Abdoulaye Diakite $^{b}$ \\ ${ }^{a}$ Department of Geoscience and Remote Sensing, Delft University of Technology, \\ Stevinweg 1, 2628 CN Delft, The Netherlands \\ ${ }^{b}$ Section 3D Geoinformation, Department of Urbanism Faculty of Architecture \\ and The Built Environment, Delft University of Technology, 2628 BL Delft, The Netherlands \\ (B.Sirmacek, Y.Shen-2, R.C.Lindenbergh, S.Zlatanova, A.A.Diakite)@ tudelft.nl
}

\section{Commission V, WG V/3}

KEY WORDS: Point Clouds, Indoor Laser Scanning, Zebedee, Leica C10

\begin{abstract}
:
We present a comparison of point cloud generation and quality of data acquired by Zebedee (Zeb1) and Leica C10 devices which are used in the same building interior. Both sensor devices come with different practical and technical advantages. As it could be expected, these advantages come with some drawbacks. Therefore, depending on the requirements of the project, it is important to have a vision about what to expect from different sensors. In this paper, we provide a detailed analysis of the point clouds of the same room interior acquired from Zeb1 and Leica C10 sensors. First, it is visually assessed how different features appear in both the Zeb1 and Leica C10 point clouds. Next, a quantitative analysis is given by comparing local point density, local noise level and stability of local normals. Finally, a simple 3D room plan is extracted from both the Zeb1 and the Leica C10 point clouds and the lengths of constructed line segments connecting corners of the room are compared. The results show that Zeb1 is far superior in ease of data acquisition. No heavy handling, hardly no measurement planning and no point cloud registration is required from the operator. The resulting point cloud has a quality in the order of centimeters, which is fine for generating a 3D interior model of a building. Our results also clearly show that fine details of for example ornaments are invisible in the Zeb1 data. If point clouds with a quality in the order of millimeters are required, still a high-end laser scanner like the Leica $\mathrm{C} 10$ is required, in combination with a more sophisticated, time-consuming and elaborative data acquisition and processing approach.
\end{abstract}

\section{INTRODUCTION}

Three-dimensional (3D) modeling of building interiors has vital importance for fields and tasks such as robotics, surveying, object scanning, mobile mapping, tourism, gaming and energy efficiency assessment. There are a number of ways to acquire 3D data, such as scanning lidar or radar, flash lidar, stereo vision, imaging sonar, and structured light triangulation, Vidas and Moghadam (2013). One of the most precise and fast 3D data acquisition methods is to use laser scanners. Even laser scanner sensors have many different technologies and designs available for generating 3D models of interiors. However, all of them come with different advantages, as well as disadvantages. The sensor devices have high variety of prices, sizes, quality, measurement capabilities and additional requirements of usage. Besides knowing that the device is affordable for the project and its measurements are satisfying for a specific application, it is also very important to consider how easy it is to make the acquisition by users and transporting the sensor device inside of the place which is going to be modeled. These important points must be considered before making a decision about which sensor device to select for a particular project. Therefore, this paper provides valuable information by focusing on practical and technical features of Zebedee (Zeb1) and Leica C10 laser scanners for interior point cloud acquisition. In Figure 1, the Zeb1 and Leica C10 Scanners are shown.

Although the Leica C10 laser scanner provides very high density and very high quality point clouds, the practical difficulties of transporting the scanner to different stations make it challenging to use in every day life, especially when the point clouds need to be generated urgently. Except for the transport of the scanner

${ }^{*}$ Corresponding author

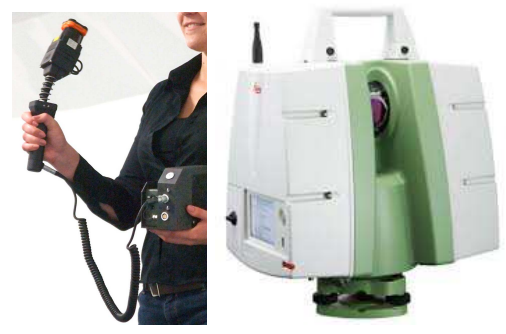

Figure 1: Left; the Zeb1 laser scanner is shown while it is being used in a scanning process. Right; the Leica C10 laser scanner.

itself during acquisition, also knowledge by the operator is required on target installation, data acquisition and post-registration steps. The issue of the different stations is solved by using a personal mobile mapping system, Lauterbach et al. (2015) and Corso and Zakhor (2013). Still, some mobile devices require a trained person to obtain a valid 3D point cloud product. The Zeb1 scanner brings advantages to overcome many of those difficulties. For instance, Zeb1 can be used by non-trained persons and the resulting point clouds can be obtained very quickly even for very large structures. As could be expected, these advantages come with some drawbacks. Zeb1 based point clouds have way less point density and precision comparing to the Leica based point clouds. When several point cloud acquisitions are done, Zeb1 data needs to be submitted to an online system for registration process. This online platform charges also payments depending on the sizes of the submitted data.

Zeb1 has been introduced by Bosse et al. (2012) as a novel design of a $3 \mathrm{D}$ range sensor consisting of a $2 \mathrm{D}$ lidar scanner mounted on a flexible spring useful for 3-D perception in robotics and other 
mobile applications. They have demonstrated a particular realization of the general design concept, which is called Zebedee A specialized SLAM algorithm (Durrant-Whyte et al. (2006)) has been developed that can be used both for incremental motion estimation and global point cloud registration, Curran et al. (2011). In fact, the same algorithm base has been used for a variety of mobile 3D laser configurations, including spinning, nodding, and trawling 2D lidars. The solution has been evaluated in a variety of environments, both indoor and outdoor, and quantitatively analyzed using a motion capture system for ground-truth position estimates. There are, however, some limitations to the system. Common to all laser-based SLAM algorithms, there exist pathological environments where the motion is weak or nonobservable, such as long, smooth tunnel-like spaces, or large, featureless, open areas. Such cases are identifiable by examining the distribution of surface normals, but extreme cases can be difficult to recover from without additional sensors. Environments dominated by moving objects can also challenge the algorithm since it becomes difficult to distinguish true outliers. In some applications, the Zeb1 design requires fairly continual excitation in order to induce motion of the sensor head. For example, the device may not be appropriate for electric ground vehicles operating with infrequent accelerations on smooth terrain. However, there are many potential excitation sources in typical applications, such as rough terrain, vibrating engines, legged platforms, wind disturbances for air vehicles or fast-moving ground vehicles, or wave motion for marine surface vehicles. It should also be noted that the trajectory computed by the SLAM solution tracks the sinuous trajectory of the sensor head: further processing would be required to also estimate the trajectory of the platform on which the sensor base is mounted. Since the compression of the spring is small compared with the bending of the spring, it behaves approximately like a rigid link with a universal joint. Thus, a simple transformation approximates the position of the sensor base frame relative to the sensor head. More accurate results can potentially be obtained by observing and tracking structure on the base platform within the local point cloud. Finally, a limitation of the SLAM solution which is presented is that it does not compute an uncertainty estimate, although there are ongoing studies on investigating this aspect.

Chane et al. (2013) used Zeb1 for 3D reconstruction of a large cultural heritage area which is a small village. Zeb1 gave the opportunity to walk in the village and generate a 3D model of the environment and the heritage buildings only in few hours. The areas which are not accessible by large devices could be modeled accurately. The 3D documentation of another cultural heritage area of $250 \times 400 \mathrm{~m}$ is discussed in Zlot et al. (2015) and the outdoor scanning of complex cliff morphology is discussed in James et al. (2014). A Zeb1 has also been discussed in Thomson et al. (2013) for the purpose of indoor mobile mapping, in Zlot et al. (2014) for the mapping of an underground mining system, while in Ryding et al. (2015), Zeb1 has been evaluated for the purpose of scanning 3D forest structure.

In a previous study, a Leica C10 laser scanner was used for 3D data acquisition of the interior and exterior of a historical windmill in Delft. The data is analyzed in the BSc thesis of Roebroeks (2015) and partly available via an open data repository, Sirmacek (2015). The exterior point cloud acquisition took few hours, however the interior point cloud acquisition of different floors and rooms took three working days. In addition to that, several working days were spent for transferring the data from the laser scanner to the computer and to correctly align the different scans. The point cloud has also color information which is not possible to obtain with the Zeb1 laser scanner. The details of the objects are very accurate and sharp. For instance, the data gives opportunity to detect deterioration or cracks on wooden panels. Measurements of distances and angles can be done at high precision. However the total windmill point cloud (together with interior and exterior points) consists of almost $60 \mathrm{~GB}$ data which is not possible to process or visualize easily on every computer. Besides these technical difficulties, another difficulty that researchers experienced was the practical phase of the data acquisition process. The laser scanner equipment was large and heavy to operate on the narrow and steep staircases of the windmill. Besides, the wood and stone based structure made it challenging to install magnetic targets or stick target papers.

In the rest of the paper, we discuss and compare point clouds of both the Leica C10 and the Zeb1 laser scanners in detail.

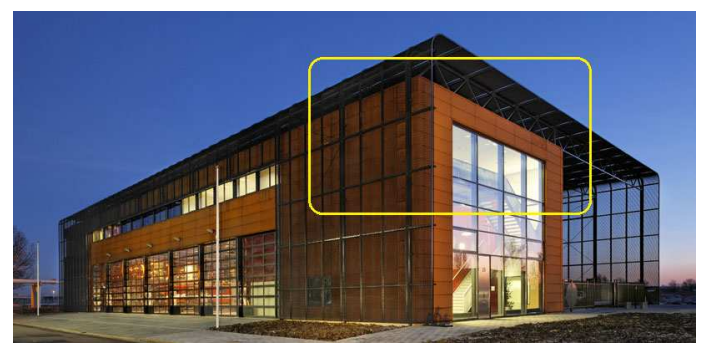

Figure 2: Outside view of the test building (Firebrigade office of Lansingerland, The Netherlands).

\section{DATA SETS, FIREBRIGADE OFFICE}

For data acquisition and comparison, we have visited the Firebrigade office of Lansingerland in the Netherlands. The outside of the building is shown in Figure 2. The yellow box highlights the position of the room which is used for detailed comparison and tests in this paper.

Because of the larger (target installation, data acquisition, postprocessing) time requirements of the Leica C10 laser scanner, we have gathered data only in a few rooms of the building. However, since the Zeb1 laser scanner can provide faster results, all building could be scanned in approximately half an hour. Figure 3 shows the path of the Zeb1 laser scanner and the resulting full building interior point cloud.

\subsection{ZEB1}

In terms of widening the appeal of scanning, probably the Zebedee is one of the most advanced devices. Peel (2014) announced it as the first truly handheld scanner. The mechanical simplicity, size, and weight of this sensor design make it suitable to develop practical solutions to a range of challenging applications, such as mapping from a micro air vehicle, or infrastructure inspection in confined spaces. A lightweight handheld or human-wearable version of a spring-mounted laser would be ideal for use as a localization or mapping tool by first responders in emergency situations. While several handheld 3-D scanners are commercially available (e.g., Z Corporations ZScanner, Leicas T-Scan, and Mantis Visions MVC-F5), they are primarily intended for object scanning applications, often require modification to the environment, and have limited working volume that is not appropriate for large-scale mobile applications. Available structured lighting solutions are limited in their sensing range, precision, and the lighting conditions in which they can operate. For example, the Microsoft Kinect has an effective range of a few meters, and a precision of around $12 \mathrm{~cm}$ at $4 \mathrm{~m}$ range, Khoshelham and Elberink (2012). 

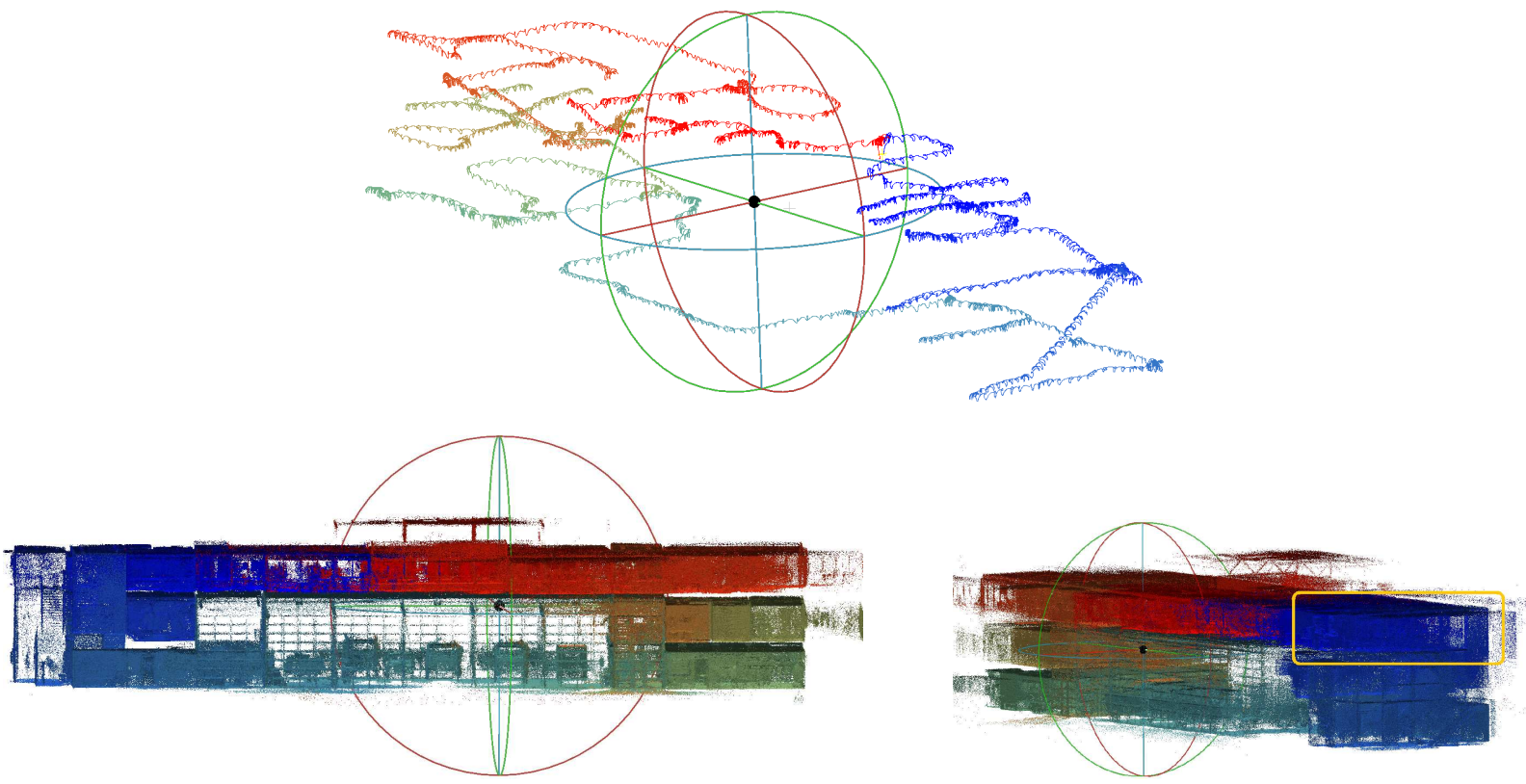

Figure 3: Top; the path of the Zeb1 sensor for the building scanning process. Bottom left; full point cloud of the building interior Bottom right; full point cloud of the building interior. All three images are false colored by time scale. The test room is highlighted in the bottom right image by a yellow box.

The Zebedee 3D sensor system consists of a 2D laser scanner and an IMU mounted on one or more springs. The laser in the current model is a Hokuyo UTM-30LX, which is a 2D time-offlight laser with a 270 degrees field of view, $30 \mathrm{~m}$ indoor and $15 \mathrm{~m}$ outdoor maximum range, and $40 \mathrm{~Hz}$ scanning rate. The dimensions of the UTM-30LX are $60 \times 60 \times 85 \mathrm{~mm}$, and its mass is $210 \mathrm{~g}$, which makes it ideal for low-weight requirements. The IMU is a MicroStrain 3DM-GX2, an industrial-grade IMU that contains triaxial MEMS gyros and accelerometers with an output rate of $100 \mathrm{~Hz}$.

\subsection{Leica C10}

The Leica C10 Scan Station scanner is a time-of-flight scanner with an effective operating range of +/- 1-200 $\mathrm{m}$ (up to $300 \mathrm{~m}$ with $90 \%$ reflectivity). It's motorized head allows scanning of a complete field of view of 360 degree by 270 degree. Data is acquired at a rate of 50,000 points/second and can be stored on-board or on a wireless or wired laptop. The $\mathrm{C} 10$ has a number of features which make it particularly effective. It has an on-board camera which can provide images to be automatically aligned with the scans to texture the point clouds. The specifications indicate that the accuracy of a single measurement is $6 \mathrm{~mm}$ in position and 4 $\mathrm{mm}$ in depth at ranges up to $50 \mathrm{~m}$, Leica (2011).

\section{QUALITY ASSESSMENT}

In this section, we focus on the laser scanning point clouds of one test room in order to discuss the capabilities of the two laser scanning sensors.

\subsection{Preparing the input data for comparison}

When after acquisition the Leica and Zeb1 point clouds are together displayed in a point cloud viewer, they initially do not match. Both input point clouds have different coordinate system that are in addition not georeferenced. There are orientation and coverage differences as well. Before starting to compare the interior scanning qualities of the two input point clouds, we need to align them correctly. To do so, we have used CloudCompare (2015) software which provides a tool for manually selecting tie points and obtain the $3 \mathrm{D}$ transformation function for aligning one of the point clouds on the other.

After aligning the Zeb1 point cloud with the Leica C10 point cloud, we have segmented walls in the room in order to be able to compare point clouds of each wall separately. The segmentation is done by extracting local normal vectors and calculating local differences. By applying a threshold to the normal vector angle differences, we have segmented the walls. Herein, we have used a normal search window of $20 \mathrm{~cm}$ and an angular difference threshold value of 45 degrees which means that surfaces having more than 45 degrees angle are segmented into different groups. More information on the algorithm is given in Ioannou et al. (2012). In Figure 4 we provide a view of the extracted wall segments for the Zeb1 and Leica C10 point clouds of test room.
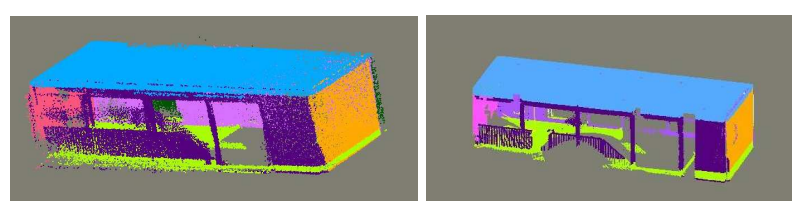

Figure 4: Left; segmented Zeb1 point cloud walls. Right; segmented Leica $\mathrm{C} 10$ point cloud walls.

\subsection{Suitability for the extraction of metric properties.}

In this section the suitability of the Leica $\mathrm{C} 10$ and the Zeb1 point cloud for metric applications are assessed. First, it is visually inspected how characteristic features in the room are represented in both point clouds. Next, a quantitative comparison is made. For this purpose the extracted wall segments in the point clouds from both sensors are used. Both wall point clouds are divided into local planar patches and their properties are compared. In 


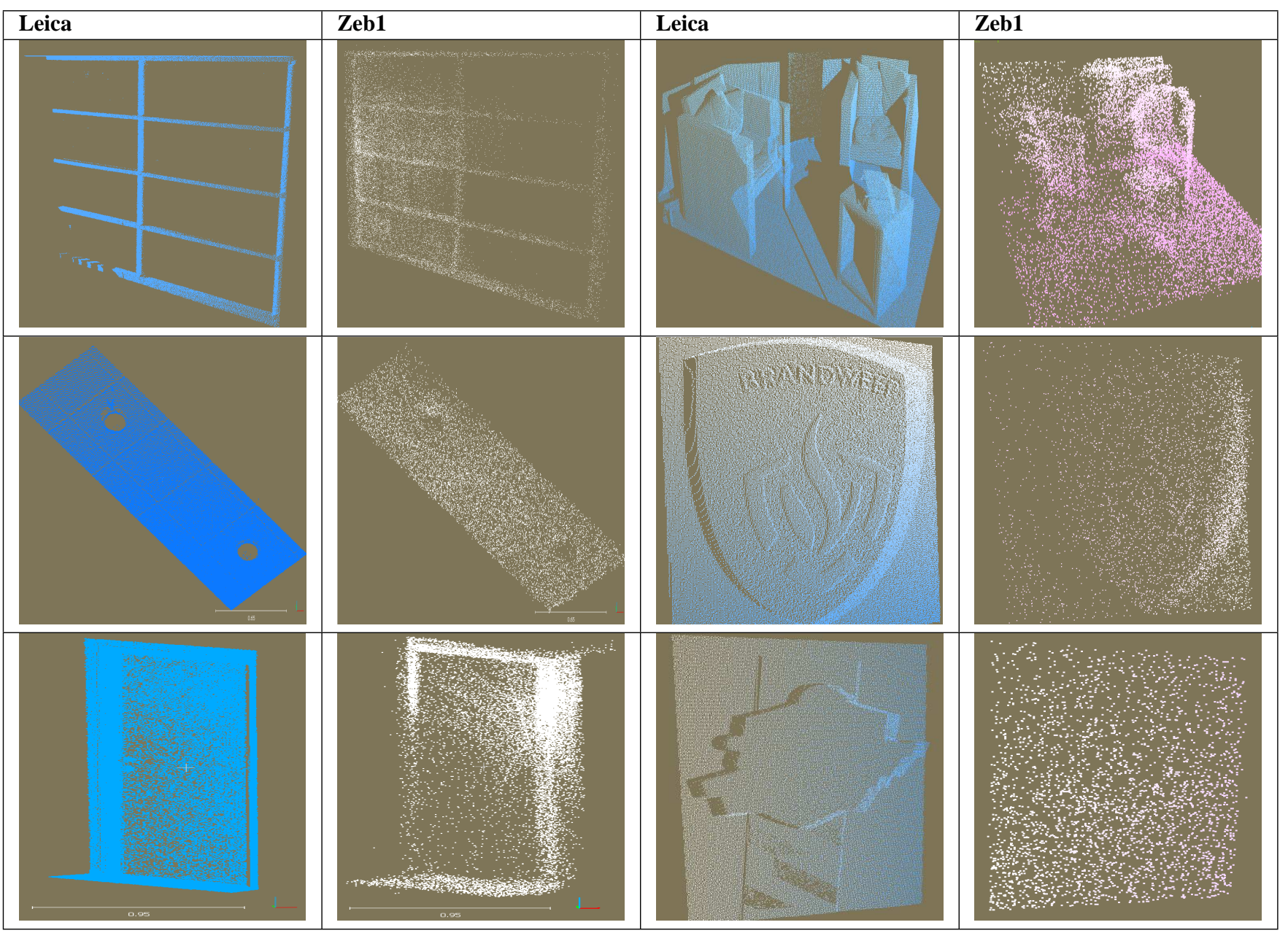

Table 1: Comparison of key objects. Leica C10 point clouds are shown in blue. The corresponding Zeb1 point cloud is shown on the right of each Leica $\mathrm{C} 10$ point cloud.

addition, distances between characteristic points like corners are extracted and compared.

3.2.1 Visual comparison. In Table 1 some of the key objects in the room are presented. Their appearance in the two different point clouds illustrate the sharpness of the 3D geometrical details and point density of the Leica $\mathrm{C} 10$ and the Zeb 1 data. From this visual inspection we conclude that coarse features like walls, are clearly visible in both point clouds. Features that vary at the decimeter scale, like chairs or cupboards are visible in both point clouds, but are already difficult to recognize in the Zeb 1 data. Finer features, like the 3D logo of the fire brigade, with variations in the order of centimeters are still sharp in the Leica C10 data but are not visible at all in the Zeb 1 data. A summary of the visibility of some key object is given in Table 2 .

3.2.2 Extracting local patches A further quantitative evaluation of the Leica $\mathrm{C} 10$ and the Zeb 1 point clouds is given based on an analysis of local planar patches. These patches are extracted and analyzed for both point clouds as follows. First an arbitrary wall is identified that is present in both point clouds. Using the segmentation results described above, all points sampling this wall for both sensors are extracted.

Next, after removing some remaining outliers, sampling for example window sills, 5000 points from each wall point cloud are randomly extracted. These 5000 points are used to estimate a global normal vector for the wall using Principal Component Analysis (PCA). The eigenvectors of the PCA are used to convert each

\begin{tabular}{|l|l|l|l|}
\hline$\#$ & Key Object & Leica & Zeb1 \\
\hline 1 & Ceiling boundaries & Visible & Not Visible \\
\hline 2 & Small wall decoration & Visible & Not Visible \\
\hline 3 & Small holes in the ceiling & Visible & Not Visible \\
\hline 4 & Staircase handles & Visible & Visible \\
\hline 5 & Window ceiling & Visible & Visible \\
\hline 6 & Larger wall decoration & Visible & Visible \\
\hline 7 & Sitting corner & Visible & Visible \\
\hline 8 & Elevator boundaries & Visible & Visible \\
\hline 9 & Door boundaries & Visible & Visible \\
\hline
\end{tabular}

Table 2: Visibility of key objects.

full wall point cloud to a $2 \mathrm{D}$ plane aligned to the wall, where the 3 rd coordinate, the $z_{i}$, gives the signed distance of the $i$-th point to this plane, compare also Van Goor et al. (2013).

The resulting 2D wall point clouds for both sensors are divided into patches of $20[\mathrm{~cm}] \times 20[\mathrm{~cm}]$. For each resulting patch the following properties are derived for both sensors:

- Local point density

- Local standard deviation

- Local deviation from global normal

The local point density is obtained by, first, counting the number of points in each patch, and, second, normalizing the result to 

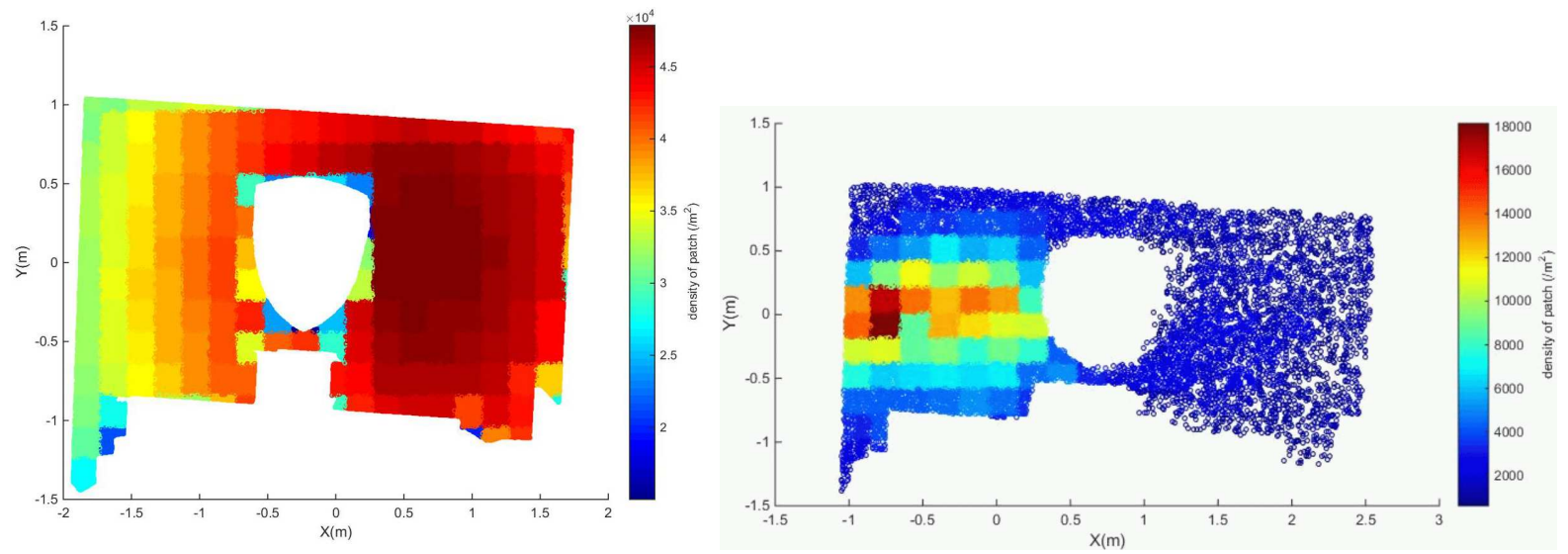

Figure 5: Local point density of patches from one of the wall segments. Left. Leica C10 point cloud. Right. Zeb1 point cloud.
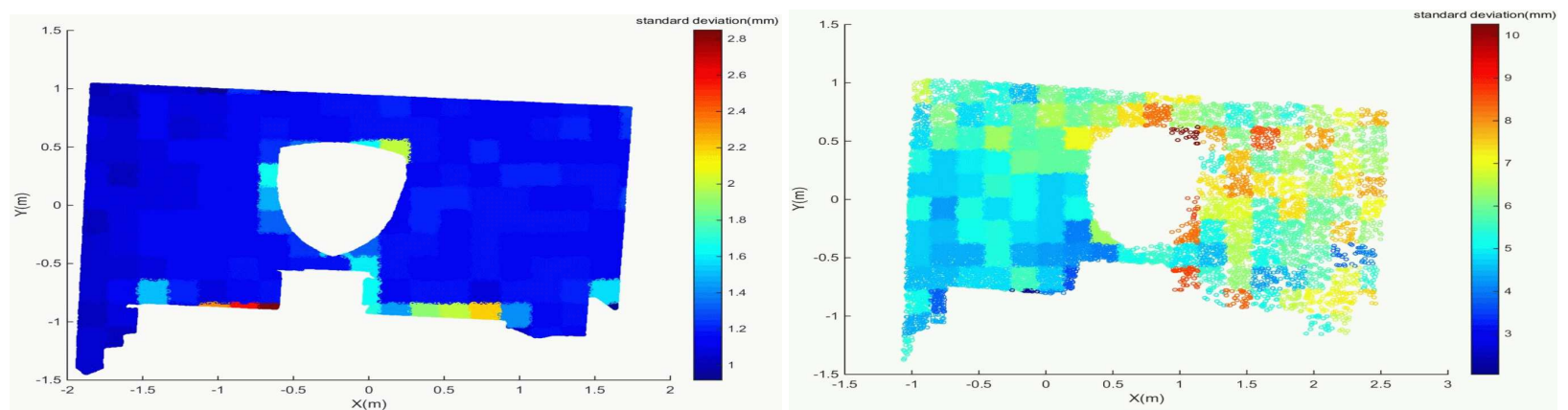

Figure 6: Standard deviation per patch for a local planar fit. Left. Leica C10 point cloud. Right. Zeb1 point cloud.
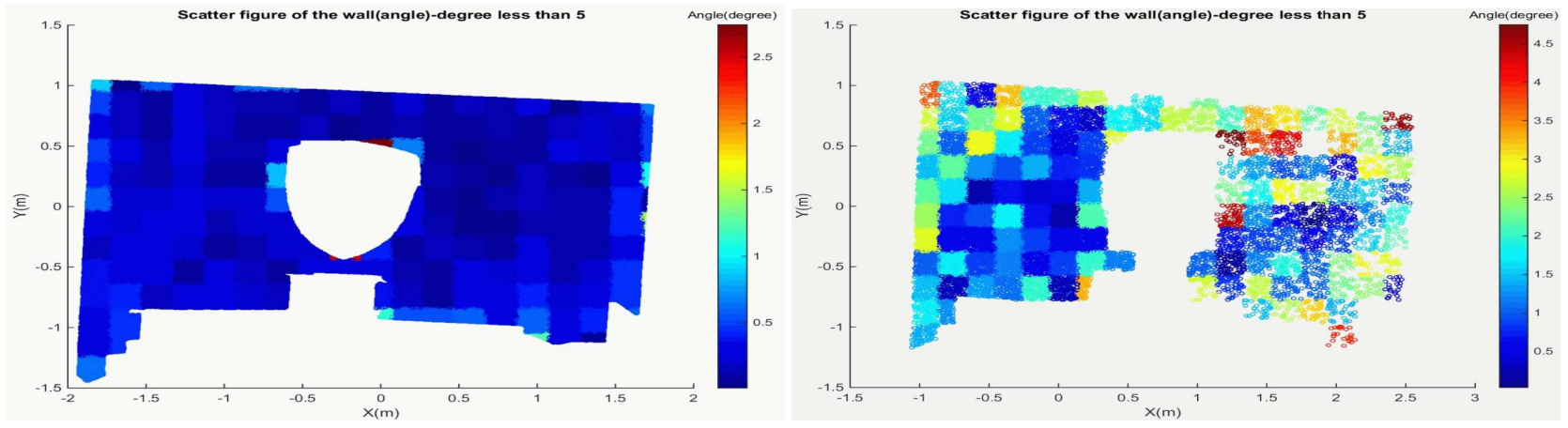

Figure 7: Difference between normal of local patch and global wall normal Left. Leica C10 point cloud. Right. Zeb1 point cloud.

the corresponding number of points per $\left[\mathrm{m}^{2}\right]$. A local standard deviation and local normal vector are obtained by a local PCA fit. The resulting local normal vector is compared to the global normal vector of the plane to arrive at a local deviation from the global normal.

3.2.3 Results, local point density The resulting local point densities for both sensors are visualized in Figure 5 by coloring point according to this point density. The point density of the Leica $\mathrm{C} 10$ point cloud sampling the wall varies between roughly 30000 points $/\left[\mathrm{m}^{2}\right]$ and roughly 50000 points $/\left[\mathrm{m}^{2}\right]$. This means that the point to point distance is in the order of $5[\mathrm{~mm}]$. Variations in the point density are caused by the relative position of the scanner w.r.t. the location of the wall during acquisition. If necessary, the point density could be further adapted by moving the scanner or changing the scan resolution.

For the Zeb 1 point cloud, individual points on the right hand side are clearly visible. For the Zeb 1 point cloud, the local point den- sity varies between 1000 points $/\left[\mathrm{m}^{2}\right]$ and 18000 points $/\left[\mathrm{m}^{2}\right]$. This means that the highest Zeb 1 point density compares to the lowest Leica C10 point density. Again, variations in point density are probably caused by the acquisition geometry. Maybe a higher point density could be obtained by for example revisiting a room. The minimal point density of 1000 points $/\left[\mathrm{m}^{2}\right]$ would correspond to a point to point distance of around $3[\mathrm{~cm}]$, which explains why some small features were not distinguishable in the above visual inspection. Still, the point densities found would probably be sufficient for most applications that consider complete buildings like considered in this work.

3.2.4 Results, local patch fitting The points in each $20[\mathrm{~cm}]$ patch were fitted to a local plane, which notably resulted in standard deviations per patch as visualized in Figure 6. The st.dev per patch for the Leica C10 data is in the order of 1-1.5 [mm]. This is a bit better then the above reported value of $4[\mathrm{~mm}]$ from the specifications, Leica (2011). A possible explanation is that these values are given for range measurements up to $50[\mathrm{~m}]$, while the 
ranges here were in the order of $5[\mathrm{~m}]$. In addition, the material and acquisition geometry will further influence the quality. In this acquisition, scanning was near perpendicular, which means that conditions were very favorable, Soudarissanane et al. (2011).

Resulting standard deviations for the Zeb 1 acquisition varied between 4 to $9[\mathrm{~mm}]$. This corresponds well to the product specification of the embedded UTM-30LX laser range finder, which states that the range st.dev is below $10[\mathrm{~mm}]$ for acquisitions up to $10[\mathrm{~m}]$, so no surprises here. But note that in this case the final quality depends both on the laser and on the IMU solution, so in that sense the Zeb 1 does a nice job, as it stays within the specs of the laser, even when the IMU solution is included.

3.2.5 Results, local normal assessment The quality of the local planar patch parameters is determined by point density and point precision. Instead of considering these parameters separately, we consider the quality of the local patch reconstruction as a whole by considering the deviation between local patch normal and global wall normal as visualized in Figure 7.

The results show that resulting local Leica $\mathrm{C} 10$ normals match the global normal up to differences that not really exceed 0.5 [deg]. The Zeb 1 local normal vary between 0.5 [deg] for the more densely sampled left part of the wall, to deviations of 3 to 4 [deg] on the more sparsely sampled right part of the wall. A first conclusion could be, that, if higher metric quality is required, acquisition by the Zeb 1 should be done such that local point density is sufficient. How this could be enforced during acquisition is not clear to this author at the time of this writing however.

\subsection{Baseline Analysis}

A final goal of a Zeb 1 acquisition could be to obtain a simple CAD map of the sampled building. For the coming analysis we assume that the Leica C10 data is superior in quality to the Zeb 1 data, which seems a reasonable assumption given the local patch analysis of above. The starting point of the coming analysis is the room segmentation for both sensors as shown before in Figure 4 .

First, planes are fitted to the segments sampling different walls. Next, by intersecting these planes, 3D locations of the eight corner points of the wall are obtained, that are visible in Figure 8 . From both the Zeb 1 data and the Leica C10 data, lengths are derived of the line segments connecting the corner points. We refer to these line segments as baselines. Finally, the lengths of the Zeb 1 baselines are compared to the corresponding Leica C10 baselines which results in certain differences in length.

In Figure 8 the baselines connecting the corner points are colored by these differences. If we assume the Leica baselines to provide ground truth, the resulting differences could give insight in the value that Zeb 1 measurements have for constructing a CAD map. The resulting differences are all between $3[\mathrm{~mm}]$ and 38 [mm]. For example, if we would like to estimate the area of a room of $6[\mathrm{~m}] \times 4[\mathrm{~m}]$ by multiplying an estimated length of 6.04 $[\mathrm{m}]$ of the room with and estimated width of $4.04[\mathrm{~m}]$, the resulting error in the estimated area would be $0.4\left[\mathrm{~m}^{2}\right]$. This worst-case scenario would be acceptable for applications such as real-estate assessment, coarse renovation planning or many cadaster measurements. If the goal of a measurement would be to estimate the size of a window sill or a glass panel for for example external construction, a higher quality is probably required.

\section{CONCLUSIONS AND FINAL REMARKS}

In this paper, we have provided comparison results of Leica and Zeb1 point clouds sampling the same room. Besides discussing practical advantages and disadvantages, we have provided detailed quantitative quality analysis in terms of local point density, noise level and reconstruction capacities. Besides, we have compared the appearance of characteristic objects in the room, like room boundaries, door handles and 3D ornaments. Finally, we have discussed the quality of a simple 3D map as extracted from the Zeb1 point cloud benchmarked against the Leica C10 point cloud. Our examples and discussions illustrate technical and practical advantages and disadvantages of two different laser scanners. We believe that our detailed comparison provides insights to select the most suitable sensor device and understand the acquired data. As future work, we are going to include Google Project Tango and Kinect based point clouds for comparison with Leica and Zeb1 point clouds. We believe that our experiments provide important information to those who use point clouds for 3D modeling, object recognition and interior navigation purposes.

\section{ACKNOWLEDGMENTS}

This research is partly funded by the NWO/STW M4S project Smart 3D indoor models to support crisis management in large public buildings (www.sims3d.net). We thank the Fire Brigade Rotterdam - Rijnmond for making one of their buildings available for the tests. The FP7 project IQmulus (FP7-ICT-2011318787) A high volume fusion and analysis platform for geospatial point clouds, coverages and volumetric data set is thanked for funding part of the co-authors. We would like to thank the China Scholarship Council for enabling Yueqian Shen to study for one year at Delft University of Technology.

\section{References}

Bosse, M., Zlot, R. and Flick, P., 2012. Zebedee: Design of a spring-mounted 3-d range sensor with application to mobile mapping. IEEE Transactions on Robotics, 28(5), pp. 1104 1119 .

Chane, C., Mansouri, A., Marzani, F. and Boochs, F., 2013. Integration of 3D and multispectral data for cultural heritage applications: Survey and perspectives Image and Vision Computing, 31(1), pp. 91-102.

CloudCompare, 2015. http://www.danielgm.net/cc/release/. Accessed: 2015-12-12

Corso, N. and Zakhor, A., 2013. Indoor Localization Algorithms for an Ambulatory Human Operated 3D Mobile Mapping System Remote Sensing, 5(12), pp. 6611-6646

Curran, K., Furey, E., Lunney, T., Santos, J., Woods, D. and Mc Caughey, A., 2011. An evaluation of indoor location determination technologies. Journal of Location Based Services, 5(2), pp. 61-78.

Durrant-Whyte, H., and Bailey, T., 2006. Simultaneous localization and mapping: part I. Robotics \& Automation Magazine, IEEE, 13(2), pp. 99-110.

Van Goor, B., Lindenbergh, R. and Soudarissanane, S., 2013. Identifying corresponding segments from repeated scan data. Int. Arch. Photogramm. Remote Sens. Spatial Inf. Sci., XXXVIII-5/W12, pp 295-300,

Ioannou, Y., Taati, B., Harrap, R. and Greenspan, M., 2012. Difference of normals as a multi-scale operator in unorganized point clouds. The Second International Conference on 3D Imaging, Modeling, Processing, Visualization and Transmission (3DIMPVT) pp. 501-508. 


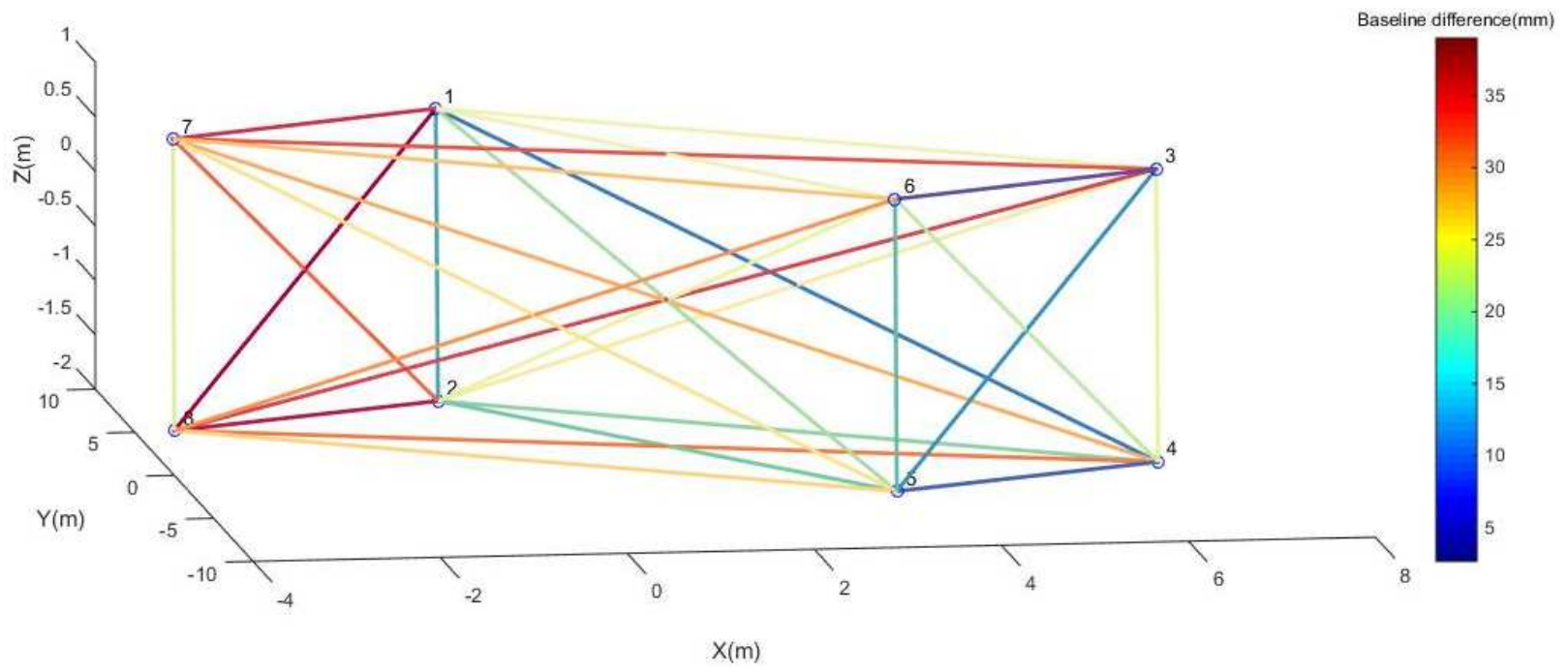

Figure 8: Visualization of differences in baselines derived from the Leica C10 point cloud and the Zeb1 point cloud..

James, M. R. and Quinton, J. N., 2014. Ultra-rapid topographic surveying for complex environments: the hand-held mobile laser scanner (HMLS). Earth Surf. Process. Landforms, 39: pp 138-142.

Khoshelham, K. and Elberink, S. O., 2012. Accuracy and resolution of kinect depth data for indoor mapping applications. Sensors, 12(2), pp 1437-1454.

Lauterbach, H. A., Borrmann, D., Heß, R., Eck, D., Schilling, K., and Nüchter, A., 2015. Evaluation of a Backpack-Mounted 3D Mobile Scanning System. Remote Sensing, 7(10), pp 13753 13781.

Leica, 2011. The all-in-one laser scanner for any application. Leica Geosystems Product Datasheet.

Peel, R., 2014. Innovation in rapid laser scan data collection and processing: The speed of light. GeoInformatics, 17(6), pp. 10 12.

Roebroeks, J., 2015. Generating a full 3d model of the windmill (molen de roos). BSc Final Thesis.

Ryding, J., Williams, E., Smith, M. J., and Eichhorn, M. P., 2015. Assessing handheld mobile laser scanners for forest surveys. Remote Sensing, 7(1), pp. 1095-1111.
Sirmacek, B. 2015. Delft Windmill Interior and Exterior Laser Scanning Point Clouds. Delft University of Technology. Dataset. http://dx.doi.org/10.4121/uuid:daea472d-2ca5-47659f1b-bd3200de4b41

Soudarissanane, S., Lindenbergh, R., Menenti, M., and Teunissen, P., 2011. Scanning geometry: Influencing factor on the quality of terrestrial laser scanning points. ISPRS Journal of Photogrammetry and Remote Sensing, 66(4), pp 389-399.

Thomson, C., Apostolopoulos, G., Backes, D. and Boehm, J., 2013. Mobile laser scanning for indoor modelling, ISPRS Ann. Photogramm. Remote Sens. Spat. Inf. Sci, 5, W2

Vidas, S. and Moghadam, P., 2013. HeatWave: A handheld 3D thermography system for energy auditing, Energy and Buildings, 66, pp 445-460.

Zlot, R. and Bosse, M., 2014. Efficient Large-scale Threedimensional Mobile Mapping for Underground Mines Journal of Field Robotics, 31(5), pp 758-779.

Zlot, R., Bosse, M., Greenop, K., Jarzab, Z., Juckes, E. and Roberts, J., 2014. Efficiently capturing large, complex cultural heritage sites with a handheld mobile 3D laser mapping system, Journal of Cultural Heritage, 15(6), pp 670-678, 